\title{
DISEÑO DE UN CUESTIONARIO PARA MEDIR LA FORMACIÓN BILINGÜE DEL PROFESORADO DE EDUCACIÓN FÍSICA [FBPEF]
}

\section{DESIGN A QUESTIONNAIRE TO MEASURE THE FORMATION \\ BILINGUAL TEACHER OF PHYSICAL EDUCATION [FBTPHED]}

Maㅡ Luisa Zagalaz Sánchez•David Molero López-Barajas • Javier Cachón Zagalaz• Universidad de Jaén [España]

Javier Gutiérrez de Castro • Profesor de Educación Primaria

Correspondencia: Izagalaz@ujaen.es [Mª Luisa Zagalaz Sánchez]

Recepción abril $2012 \cdot$ Aceptación junio 2012

\section{RESUMEN}

El artículo recoge el diseño de un instrumento para medir la opinión del profesorado de Educación Física (EF) en Educación Primaria (EP) sobre la enseñanza bilingüe (inglés-español) de su materia, es decir, un cuestionario que permita conocer si el profesorado se siente capacitado para impartir clases de EF en inglés.

La metodología seguida se encuadra dentro del paradigma positivista mediante un estudio descriptivo. El instrumento objeto de estudio supone una buena aproximación como medida.

El cuestionario se aplicó a una muestra de 40 sujetos, todos ellos con la titulación Diplomado en EF, para posteriormente proceder a la obtención de la fiabilidad del instrumento previamente diseñado. Calculamos el coeficiente alfa de Cronbach (1951) que fue para el conjunto del cuestionario de 0,83 .

No obstante son necesarias más investigaciones que profundicen cualitativamente para complementar los resultados.

Palabras clave: Educación Física, bilingüismo, inglés, educación primaria.

\section{ABSTRACT}

The article gathers the design of an instrument to measure the opinion of the professorship of Physical Education (Ph.Ed.) in Primary Education (P.Ed.) on the bilingual education (English-Spanish) of his matter, that is to say, a questionnaire that allows to know if the professorship feels qualified to give EF's classes in English.

The followed methodology is fitted inside the positivist paradigm by means of a descriptive study. The instrument object of study supposes a good approximation as measure.

The questionnaire graduate applied himself to a sample of 40 subjects, all of them with the qualifications in $\mathrm{Ph}$.Ed., later to proceed to the obtaining of the reliability of the before designed instrument. We calculate the coefficient alpha of Cronbach (1951) that was for the set of the questionnaire of 0.83 .

Nevertheless there are necessary more investigations that penetrate qualitatively to complement the results.

Key Words: Physical education, bilingualism, English, primary education.

\section{INTRODUCCIÓN}

Antes de comenzar el desarrollo de este artículo, conviene llevar a cabo una definición de educación o enseñanza bilingüe, que de acuerdo con Siguán \& Mackey (1986, p. 62) sería un sistema educativo en el que se utilizan dos lenguas como medio de instrucción, de las cuales normalmente, aunque no siempre, una es la primera lengua de los alumnos o lengua materna.

Son muchas las investigaciones que se han llevado a cabo a lo largo del tiempo en relación al bilingüismo en general, y distintos autores los que han intentado demostrar a través de diversos estudios la diferencia entre personas bilingües y monolingües con respecto a su funcionamiento cognitivo. Sin embargo es muy poco lo investigado sobre la relación entre el bilingüismo y la EF para mejorar el aprendizaje de una segunda lengua, destacando el estudio de D'Acierno (2011, p. 27) entre niños y adultos sobre la importancia de la interdisciplinariedad entre el deporte, la música y el aprendizaje de otra lengua, que concluye que la coordinación mental y física del movimiento apoyado en la música mejora el aprendizaje de una lengua extranjera ya que contribuye a mejorar el cuerpo y a estimular la cognición.

También Torres Otón (2010), trata de medir si el deporte y la enseñanza de práctica deportiva son un aliado para promover, entre otras cuestiones, la mejora de la expresión oral en el aprendizaje de nuevas lenguas, para lo que desarrolla una experiencia en la Universidad de Hanoi (Vietnam) a través de una cooperación entre el lectorado de español de ésta y un doctorado de la Universidad de Santiago de Compostela incorporando unas sesiones de práctica deportiva a cargo de un titulado en EF en la materia de Lengua
Española I de la Titulación de Filología Española y demostrando la efectividad del proyecto

Según Baker (1997, p. 158), desde principios del siglo XIX hasta aproximadamente los años sesenta del siglo pasado, la creencia dominante entre los investigadores era que el bilingüismo tenía un efecto perjudicial sobre el pensamiento y el desarrollo cognitivo. Opina que un momento decisivo en la historia de la relación entre el bilingüismo y cognición fue alcanzado en la investigación de Peal \& Lambert (1962), ya que es una muestra de indagación que anunció el enfoque moderno sobre el bilingüismo y funcionamiento cognitivo, es decir, encontraron pruebas de que el bilingüismo no tiene necesariamente que tener consecuencias perjudiciales o ni si quiera neutrales, más bien, existe posibilidad de que el bilingüismo lleve ventajas cognitivas sobre el monolingüismo, a parte de la importancia social actual de manejar dos lenguas para optar al mercado laboral.

Por otro lado, debido a la creciente necesidad por parte de las autoridades educativas de España, en particular de Andalucía, de incrementar el nivel y la formación del alumnado en todas las etapas de educación obligatoria, en cuanto a la primera lengua extranjera se refiere, en nuestro caso el inglés, se está llevando a cabo desde 2005 el Plan de Fomento del Plurilingüismo, un proyecto para la creación de más de 400 centros bilingües, con el objetivo de formar alumnos bilingües desde el primer ciclo de EP, algo que se vislumbra de difícil consecución cuando ya han pasado siete años.

Sobre ello, López del Castillo (2008) opina que la política lingüística que se está instaurando en la mayoría de los centros 
educativos a través del Plan de Fomento del Plurilingüismo rompe con la tradicional separación que se lleva a cabo en la enseñanzaaprendizaje de las lenguas y, en este sentido, ha propuesto un nuevo modelo curricular, el currículo integrado, para todas las lenguas, materna o extranjeras, y para todas las etapas y modalidades educativas que englobe, además de los contenidos lingüísticos que han de figurar en los currículos de lenguas, y en otras competencias cognitivas.

Desde el área de EF debemos contribuir a la mejora de las competencias tanto en la primera como en la segunda lengua, mediante el desarrollo de competencias metalingüísticas, ya que la adquisición de una segunda lengua supone aprender el idioma en situaciones comunes y la $\mathrm{EF}$ es una de las áreas que permite aprender y poner en práctica un segundo idioma en este tipo de situaciones. Especialmente cuando en el ámbito deportivo se manejan muchas palabras en inglés que los niños conocen sin problema.

Entendemos que hay que concebir la enseñanza-aprendizaje de la lengua extranjera como un proceso de participación guiada, lo que requiere una didáctica o metodología en la que se combina el traspaso de responsabilidad del profesorado al alumnado con el uso instrumental de dicha lengua. Es por ello que la opinión del profesorado al respecto es de suma importancia.

\section{OBJETIVO}

En este contexto, surge la idea de desarrollar una investigación que tiene como objetivo principal la creación de un cuestionario capaz de medir válida y fiablemente si los docentes de EF en EP están capacitados para impartir su materia con un método bilingüe.

\section{MÉTODO}

El procedimiento que se ha llevado a cabo para la creación del cuestionario, ha estado dividido en distintos pasos, que se detallan a continuación:

En primer lugar se decidió acometer este estudio mediante la aplicación de una encuesta en grupo de respuestas cerradas que es a su vez escrita, individual y estandarizada, es decir, utilizaremos un cuestionario como instrumento de recopilación de datos.

A continuación se realizó una revisión de la literatura, para comprobar la existencia de algún cuestionario similar al propósito de nuestro instrumento y comprobar si se había estudiado previamente la capacitación del profesorado de EF para la enseñanza de su área de forma bilingüe. Se revisaron bases de datos como: Teseo, Eric, Medline, Dialnet, así como distintos autores representativos en temas de bilingüismo, educación y EF como: Verdú \& Yvette (2002); Soto (2009); Paulston (1992); Siguan (2002); o Zagalaz \& Cachón (2009).

Como no se encontró ningún trabajo que midiese lo mismo que buscábamos ni pudiera ser adaptado a nuestros intereses o a nuestro contexto, pasamos a documentarnos en la forma de construir cuestionarios utilizados en la investigación educativa. Las etapas para conseguir la estructura del cuestionario, parten del objetivo general de nuestro estudio que como hemos mencionado antes consiste en conocer la opinión del profesorado de EF en EP con respecto a la enseñanza bilingüe de su materia; y las seis dimensiones que abarcaran los aspectos que queríamos conocer. Las dimensiones recogen los aspectos relacionados con:

1. La asignatura de inglés.

2. La asignatura de Educación Física.

3. El alumnado de Educación Primaria.

4. Los docentes de Educación Física.

5. El resto de los docentes.

6. Los Centros educativos y las leyes de Educación.

Cabe destacar, que con el establecimiento de estas seis dimensiones, se pretende abarcar todas las perspectivas que queremos medir con el cuestionario y que se desglosarán en los correspondientes ítems. Dichos aspectos son:
Con las dos primeras dimensiones, que están relacionadas directamente con las materias de inglés y de EF, se pretende abarcar todos los aspectos referidos a las dos áreas de conocimiento en las que se sustenta el cuestionario, es decir EF e inglés, como por ejemplo los aspectos metodológicos de ambas, las posibilidades de actuación con respecto a la EF bilingüe o los intereses de cada una de las materias.

Con las tres siguientes que recogen aspectos relacionados con los docentes de EF y resto de áreas, además del alumnado, queremos conseguir reflejar las dos partes fundamentales en las que recae la educación (docente y alumnado) comprobando la formación, intereses, experiencias y aspectos futuros de ambos.

En cuanto a la última dimensión, referida a las características relacionadas con los centros y leyes de educación, buscamos la opinión de los docentes en cuanto a la capacitación o no por parte de los centros y leyes para llevar a cabo o no una metodología de enseñanza bilingüe.

Tras establecer las seis dimensiones que forman el cuestionario y la población objeto de aplicación (los docentes de EF en EP) nos planteamos el formato de respuesta de los ítems, en nuestro caso, una escala de valoración tipo Likert, con todas las características que conlleva según Lozano \& de la Fuente (2009):

- Solo se puede preguntar un aspecto en cada ítem.

- La redacción de las preguntas tiene que ser clara y concisa.

- El enunciado debe ser coherente con el formato respuesta.

- Las alternativas de respuesta han de ser equidistantes.

Asimismo y aunque existen muchas investigaciones donde se debate cual es el número de alternativas de respuesta más adecuado a la hora de elaborar una escala tipo Likert, tuvimos en cuenta a Lozano (2004) y Lozano, García-Cueto \& Muñiz (2008) que aconsejan entre cinco y siete alternativas, optando en nuestro caso por cinco.

Para redactar los ítems, utilizamos la tormenta de ideas entre los investigadores y profesorado de EP y las motivaciones personales para acometer este estudio de todos los autores. Asimismo se recabó información del alumnado de magisterio de la UJA de las especialidades de EF e inglés que nos permitiera establecer ítems coherentes con la búsqueda que tratamos de realizar.

Se establecieron en un principio 34 items quedando al final una suma total de 30 ítems, relacionados con las distintas dimensiones aceptadas con anterioridad.

\section{RESULTADOS}

Una vez elaborado el universo de ítems, se procedió a realizar el estudio de validez, realizado desde una perspectiva teórica y otra empírica, en ambas se ha comprobado que el instrumento es válido porque sirve para medir aquello para lo que fue construido. Para la primera se ha recurrido a la aplicación de un equipo de expertos y al pretest. Para la segunda se ha utilizado el procedimiento de análisis factorial confirmatorio. Es necesario señalar que, ante la escasez de instrumentos similares reconocidos por la comunidad científica (ad hoc), no es posible validar el nuestro de forma adecuada por procedimientos de comparación ni correlación (Mateo, 1997, citado por Zagalaz, 2007, p. 200). Por tanto, la validez que aquí se presenta se refiere tan solo a la de constructo, con la cual es posible valorar si la estructura teórica del instrumento queda respaldada por la articulación real de los datos.

Para obtener la validez teórica, recurrimos en primer lugar a la valoración por jueces expertos, docentes e investigadores de distintas universidades españolas y sudamericanas, a quienes se les pidió que dieran sus aportaciones en cuanto a la calidad de redacción de los ítems, su permanencia o eliminación y la posibilidad de incluir alguno más (valoración cualitativa).

Para la valoración cuantitativa, se realizó la media de las respuestas, ítem a ítem, teniendo en cuenta todas las medias iguales a tres o por encima, como ítem a considerar y las medias por debajo de tres como ítem a modificar. Se encontraron cuatro medias de los ítems evaluados por los jueces expertos inferiores a tres por lo que optamos por su 
eliminación, el resto fueron superiores a tres, además hubo aportaciones cualitativas con respecto al cuestionario, las más significativas fueron las siguientes:

- Utilizar un lenguaje coeducativo en todo lo que se refiere a la redacción de cada una de las seis dimensiones y de los distintos ítems. Referirse a los dos géneros tanto en término de docentes como de alumnado.

- No mezclar la terminología entre la enseñanza de un idioma extranjero o segunda lengua y una terminología referida a una educación con una metodología bilingüe, por tanto utilizar siempre el mismo criterio.

- Modificar distintos ítem, para mayor comprensión por parte de los docentes que lo realicen cuando se lleve a cabo la investigación que se pretende.

Finalizados los pasos expuestos y una vez modificados todo aquellos aspectos plateados por los jueces expertos, se aplicó el cuestionario a los sujetos de una muestra, a modo de estudio piloto o pretest $(\mathrm{N}=40)$, todos ellos con al menos la titulación de Maestro/a especialista en EF, de los que 30 (75\%) eran hombres y $10(25 \%)$ mujeres.

A continuación y para medir la validez empírica o de constructo del instrumento realizamos un análisis de fiabilidad, prueba que nos permite comprobar la precisión y exactitud del cuestionario. Se calculó la consistencia interna entre todos los ítems que lo componen para saber hasta qué punto todas estas cuestiones están midiendo lo mismo (lo que tratamos de medir). Para ello es necesario, según Cronbach (1951), calcular el coeficiente alfa (_), que para el conjunto del cuestionario es de 0,83 , quedando los valores de fiabilidad para cada una de las dimensiones consideradas de la siguiente manera:

En la tabla 1 se pueden apreciar los resultados de fiabilidad obtenidos para todo el cuestionario en conjunto, como los valores de cada una de las distintas dimensiones que componen el cuestionario.

Una vez que hemos comprobado la fiabilidad del cuestionario, pasamos a calcular la validez de constructo. Hemos calculado la validez factorial, utilizando la técnica estadística del Análisis Factorial Confirmatorio, que se utiliza para agrupar los ítems en función de sus correlaciones, es decir, se puede afirmar que un test tiene validez factorial cuando al observar los ítems elaborados para medir una dimensión se agrupan diferenciándose de otros destinados a evaluar otro aspecto diferente del mismo constructo.

En la tabla 2 se pueden apreciar los resultados obtenidos en la medida de adecuación muestral KMO y los resultados de la prueba de esfericidad de Bartlett.

Se han obtenido seis factores que explican el 64,2\% de la varianza, cuyo tanto por ciento explicado por cada factor es la siguiente:

- $1^{\circ}$ Factor explica un 21, 3\% de la varianza.

- $2^{\circ}$ Factor explica un $16,3 \%$ de la varianza.

- $3^{\circ}$ Factor explica un 8,2\% de la varianza.

- $4^{\circ}$ Factor explica un 7,24\% de la varianza.

- $5^{\circ}$ Factor explica un 5,57\% de la varianza.

- $6^{\circ}$ Factor explica un 5,46\% de la varianza.

Una vez que se han llevado a cabo todos los trámites y análisis que se han expuesto en los apartados anteriores, se elaboró la versión final del cuestionario, en función de los resultados obtenidos en el Análisis Factorial confirmatorio (AFC) del mismo (Anexo 1).

\section{CONCLUSIONES}

Las seis dimensiones abarcan los aspectos que queremos medir, engloban las partes fundamentales en las que recae la educación y permiten obtener la opinión de los docentes de EF en cuanto a la capacitación o no por parte de los centros y las leyes de Educación para llevar a cabo una metodología bilingüe. Por lo que el cuestionario (FBPEF) presenta precisión y exactitud con respecto al objetivo marcado.

A modo de análisis DAFO (Debilidades, Amenazas, Fortalezas y Oportunidades), podríamos plantear las siguientes conclusiones:

Fortalezas: El resultado final en cuanto al análisis de fiabilidad se refiere, que nos permite comprobar la precisión y exactitud con la que mide el cuestionario, es un resultado amplio. El coeficiente alfa fue de $0,83(=0,83)$.

Las valoraciones y aportaciones de los jueces expertos, basadas en la aceptación o rechazo de cada uno de los ítems, así como los aspectos comentados por ellos han sido positivas. Se trata de un cuestionario válido y fiable para poder utilizarlo en cualquier investigación científica en el ámbito de la educación.

Debilidades: Para futuras investigaciones, se recomienda recabar información de tipo cualitativo como complemento a los aspectos cuantitativos recogidos por el cuestionario.

Tabla 1: Fiabilidad del cuestionario (_ de Cronbach)

\begin{tabular}{|l|c|}
\hline Dimensiones & Alfa de Cronbach (_) \\
\hline Fiabilidad total del cuestionario & 0.838 \\
\hline Dimensión I: Aspectos relacionados con la asignatura de Inglés. & 0.559 \\
\hline Dimensión II: Aspectos relacionados con la asignatura de Educación Física. & 0.339 \\
\hline Dimensión III: Aspectos relacionados con el alumnado. & 0.589 \\
\hline Dimensión IV: Aspectos relevantes a los docentes de Educación Física. & 0.603 \\
\hline Dimensión V: Aspectos relevantes al resto de los Docentes. & 0.755 \\
\hline Dimensión VI: Aspectos relevantes a los centros y leyes de Educación. & 0.604 \\
\hline
\end{tabular}

Tabla 2: KMO y prueba de Bartlett

\begin{tabular}{|l|c|}
\hline Medida de adecuación muestral de Kaiser-Meyer-Olkin (KMO) & 0.249 \\
\hline \multicolumn{1}{|c|}{ Prueba de esfericidad de Bartlett } \\
\hline Chi-cuadrado aproximado & 752.874 \\
\hline Gl & 435 \\
\hline Sig & 0.000 \\
\hline
\end{tabular}


Oportunidades: Es un momento decisivo para acometer este tipo de estudios y como consecuencia de los resultados obtenidos, realizar intervenciones educativas en los ámbitos de EF e idioma extranjero (inglés).

Amenazas: Posibilidades de que los planes de bilingüismo no se lleven a cabo con el rigor necesario que puede abocar en el problema del aprendizaje del inglés en nuestro país del que todos se hacen eco.

\section{REFERENCIAS BIBLIOGRÁFICAS}

Baker, C. (1997). Fundamentos de Educación bilingüe y bilingüismo. Madrid: Editorial Catédra.

Consejo de Europa (2002). Marco Común europeo de referencia para las lenguas: aprendizaje, enseñanza, evaluación. http://cvc.cervantes.es/obref/marco.

Cronbach, L.J. (1951). Coefficient alpha and internal structure of test. Psychometrika, 12, 1-16.

D'Acierno, M.R. (2011). Sport, Music and Foreign Languages. Abstract Book for the 7th Annual International Conference on Kinesiology \& Exercise Sciences, 27-30 June, 2011, Athens, Greece, 27-28. López del Castillo, C. (2008). La Educación Física y el bilingüismo. Practica deporte y aprende inglés. Revista Digital Educación y Futuro, $1-5$.

http:/ / www.cesdonbosco.com/revista/articulos 2008/mayo08/m $\%$ C $2 \%$ AAcarmen.pdf

Lozano, L.M., \& De la Fuente, I. (2009). Diseño y validación de cuestionarios. Capítulo 9. En A. Pantoja, Manual básico para la realización de tesinas, tesis y trabajos de investigación. Madrid: EOS, 251-274.

Lozano, L.M. (2004). Efectos en el número de alternativas en las propiedades psicométricas de las escalas tipo Likert. Oviedo: Universidad de Oviedo.

Lozano, L.M., García-Cueto, E., \& Muñiz, J. (2008). Effect of the number of response categories on the reliability and validity of ratings cales. Methodology, 4, 73-74.

Mateo, J. (1997). La investigación ex-post-facto. Barcelona: Ediuoc. Peal, E., \& Lambert, W.E. (1962). The relationship of bilingualism to intelligence. Psychological Monographs, 76, 1-23.

Plan de Fomento del Plurilingüismo (2005). Una política para la sociedad andaluza. Sevilla: Consejería de Educación. Junta de Andalucía.

Paulston, D.P. (1992). Sociolinguistic perspectives on bilingual education. Clevendon: Multilingual matters.

Siguan, M., \& Mackey, W.F. (1986). Éducation et bilinguisme. París: UNESCO. Lauzanna: Delachaux \& Niestlé.

Siguan, M. (2001). Bilingüismo y lenguas en contacto. Madrid: Alianza ensayo.

Soto, C. (2009). Inglés como lengua extranjera. Capítulo 4. Desarrollo cultural en la enseñanza básica. La mediación del profesorado a través de las distintas áreas. Jaén: Editorial Logoss, 75-95.

Torres, A.M. (2010). La práctica deportiva como instrumento para el aprendizaje de una lengua extranjera. III Congreso Internacional de Ciencias del Deporte y Educación Física. Pontevedra, 6-8 de Mayo de 2010. https://www.altorendimiento.com/es/congresos/educacionfisica

Verdú, M., \& Yvette, C. (2002). La enseñanza del inglés en el aula de primaria: propuesta para el diseño de unidades didácticas. Murcia: Universidad de Murcia.

Zagalaz, M.L. (2007). Valoración de la educación física escolar por el alumnado de educación primaria. Estudio en la provincia de Jaén. Proyecto de investigación para optar a la CU de Didáctica de la expresión corporal. Universidad de Jaén.

Zagalaz, M.L., \& Cachón, J. (2009). Educación Física. Capítulo 9. En M.L. Zagalaz, J. Cachón \& A. Lara (Eds.), Desarrollo cultural en la enseñanza básica. La mediación del profesorado a través de las distintas áreas (pp. 181-197). Jaén: Editorial Logoss.

\section{ANEXO}

Cuestionario sobre la formación bilingüe del profesorado de Educación Física (FBPEF)

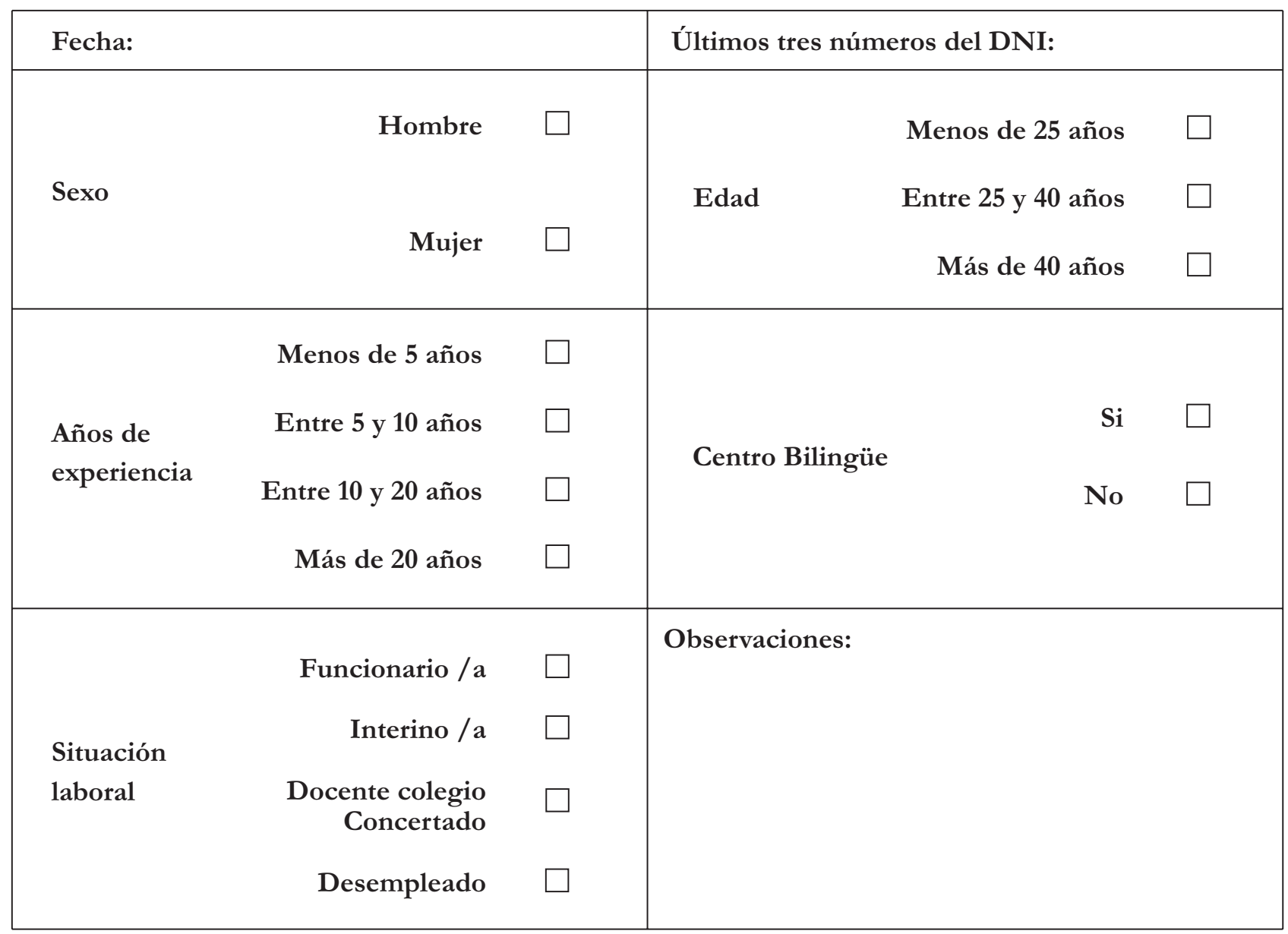


Lea atentamente cada ítem y señale el valor que crea que le corresponde atendiendo a lo que se señala en el primer cuadrante.

\begin{tabular}{|c|c|c|c|c|}
\hline 1 & 2 & 3 & 4 & 5 \\
\hline MUY POCO & POCO & ALGO & BASTANTE & MUCHO \\
\hline
\end{tabular}

\begin{tabular}{|c|c|c|c|c|c|c|}
\hline 1 & $\begin{array}{l}\text { ¿Qué importancia le da al idioma (inglés) dentro de la enseñanza } \\
\text { obligatoria? }\end{array}$ & 1 & 2 & 3 & 4 & 5 \\
\hline 2 & $\begin{array}{l}\text { ¿Qué importancia tiene para usted la adquisición del manejo del } \\
\text { idioma extranjero por parte del alumnado de cara a su formación } \\
\text { global? }\end{array}$ & 1 & 2 & 3 & 4 & 5 \\
\hline 3 & $\begin{array}{l}\text { En qué medida opina que se puede relacionar la enseñanza del idioma } \\
\text { extranjero con la de Educación Física, en cuanto a los objetivos, } \\
\text { contenido y metodología se refiere }\end{array}$ & 1 & 2 & 3 & 4 & 5 \\
\hline 4 & ¿Qué importancia le da a la enseñanza bilingüe en Primaria? & 1 & 2 & 3 & 4 & 5 \\
\hline 5 & $\begin{array}{l}\text { ¿Piensa que con la educación bilingüe se puede mejorar el nivel de } \\
\text { inglés del alumnado? }\end{array}$ & 1 & 2 & 3 & 4 & 5 \\
\hline 6 & $\begin{array}{l}\text { ¿Cree que está bien estructurada la asignatura de inglés en Educación } \\
\text { Primaria? }\end{array}$ & 1 & 2 & 3 & 4 & 5 \\
\hline 7 & $\begin{array}{l}\text { ¿Qué importancia le da a la Educación Física en el ámbito de la } \\
\text { Educación bilingüe? }\end{array}$ & 1 & 2 & 3 & 4 & 5 \\
\hline 8 & $\begin{array}{l}\text { ¿Cree que la Educación Física tiene cualidades o ventajas con respecto } \\
\text { a otras asignaturas para llevarla a cabo en un marco bilingüe? }\end{array}$ & 1 & 2 & 3 & 4 & 5 \\
\hline 9 & $\begin{array}{l}\text { ¿Piensa que la Educación Física tiene desventajas con respecto a otras } \\
\text { materias para llevarla a cabo en un marco bilingüe? }\end{array}$ & 1 & 2 & 3 & 4 & 5 \\
\hline 10 & $\begin{array}{l}\text { ¿Opina que se puede adaptar la enseñanza actual en Educación física } \\
\text { de una manera bilingüe? }\end{array}$ & 1 & 2 & 3 & 4 & 5 \\
\hline 11 & $\begin{array}{l}\text { Señale en que medida la enseñanza del idioma extranjero se relaciona } \\
\text { con la Educación Física }\end{array}$ & 1 & 2 & 3 & 4 & 5 \\
\hline 12 & $\begin{array}{l}\text { ¿Qué experiencia cree que obtiene el alumnado recibiendo una } \\
\text { enseñanza bilingüe en Educación Física? }\end{array}$ & 1 & 2 & 3 & 4 & 5 \\
\hline 13 & $\begin{array}{l}\text { ¿Qué importancia le da usted que el alumnado acabe la etapa de } \\
\text { Primaria con un manejo del idioma extranjero adecuado según los } \\
\text { objetivos buscados? }\end{array}$ & 1 & 2 & 3 & 4 & 5 \\
\hline 14 & $\begin{array}{l}\text { ¿Piensa que el alumnado está preparado y capacitado para recibir la } \\
\text { clase de Educación Física en inglés? }\end{array}$ & 1 & 2 & 3 & 4 & 5 \\
\hline 15 & $\begin{array}{l}\text { ¿Piensa que gracias a la enseñanza bilingüe el alumnado puede aumentar } \\
\text { su manejo y nivel de la lengua inglesa? }\end{array}$ & 1 & 2 & 3 & 4 & 5 \\
\hline 16 & $\begin{array}{l}\text { ¿Piensa que el alumnado adquirirá mayor motivación a la hora del } \\
\text { aprendizaje del idioma extranjero si se trabaja también en Educación } \\
\text { Física? }\end{array}$ & 1 & 2 & 3 & 4 & 5 \\
\hline 17 & $\begin{array}{l}\text { ¿Qué experiencia cree que tiene el alumnado recibiendo una enseñanza } \\
\text { bilingüe? }\end{array}$ & 1 & 2 & 3 & 4 & 5 \\
\hline 18 & ¿Se considera preparado para impartir enseñanza bilingüe? & 1 & 2 & 3 & 4 & 5 \\
\hline
\end{tabular}




\begin{tabular}{|c|c|c|c|c|c|c|}
\hline 19 & $\begin{array}{l}\text { ¿Piensa que los docentes de Educación Física deberían ser sometidos } \\
\text { a unas pruebas de nivel, que habrá que superar para acometer una } \\
\text { enseñanza Bilingüe? }\end{array}$ & 1 & 2 & 3 & 4 & 5 \\
\hline 20 & $\begin{array}{l}\text { ¿Qué experiencia tiene impartiendo Educación Física con una } \\
\text { metodología bilingüe? }\end{array}$ & 1 & 2 & 3 & 4 & 5 \\
\hline 21 & $\begin{array}{l}\text { ¿Piensa que el profesorado tendrá mayor motivación a la hora de } \\
\text { impartir Educación Física si es de una manera bilingüe? }\end{array}$ & 1 & 2 & 3 & 4 & 5 \\
\hline 22 & $\begin{array}{l}\text { ¿Qué nivel considera que tiene el resto del profesorado especialista, } \\
\text { en cuanto a la enseñanza bilingüe en Educación física? }\end{array}$ & 1 & 2 & 3 & 4 & 5 \\
\hline 23 & $\begin{array}{l}\text { ¿Estaría dispuesta/o a realizar cursos y aplicarlos para mejorar su } \\
\text { capacitación para la enseñanza bilingüe? }\end{array}$ & 1 & 2 & 3 & 4 & 5 \\
\hline 24 & $\begin{array}{l}\text { ¿Considera igual de capacitados a los docentes de inglés que a los } \\
\text { docentes de Educación Física para impartir enseñanza bilingüe? }\end{array}$ & 1 & 2 & 3 & 4 & 5 \\
\hline 25 & $\begin{array}{l}\text { ¿Qué nivel considera que tiene el resto de sus compañeros/as de } \\
\text { otras materias (menos lengua extranjera) para llevarlas a cabo con } \\
\text { una metodología bilingüe? }\end{array}$ & 1 & 2 & 3 & 4 & 5 \\
\hline 26 & $\begin{array}{l}¿ \text { Con que preparación piensa que llegan los docentes recién egresados } \\
\text { en cuanto a la enseñanza bilingüe? }\end{array}$ & 1 & 2 & 3 & 4 & 5 \\
\hline 27 & $\begin{array}{l}\text { ¿Piensa que desde la Universidad se está formando al alumnado de } \\
\text { Educación Física para la puesta en práctica de una enseñanza bilingüe? }\end{array}$ & 1 & 2 & 3 & 4 & 5 \\
\hline 28 & $\begin{array}{l}\text { ¿Piensa que los centros están capacitados en cuanto a materiales o } \\
\text { instrumentos para la enseñanza bilingüe? }\end{array}$ & 1 & 2 & 3 & 4 & 5 \\
\hline 29 & ¿Conoce metodologías bilingües y su puesta en práctica? & 1 & 2 & 3 & 4 & 5 \\
\hline 30 & ¿Modificaría las leyes de Educación para implantar un modelo bilingüe & 1 & 2 & 3 & 4 & 5 \\
\hline
\end{tabular}

